\title{
Platonic Gaussian beams: wave and ray treatment
}

\author{
R. Gutiérrez-Cuevas ${ }^{1, *}$ (1) And M. A. Alonso ${ }^{1,2,3}$ (i) \\ ${ }^{1}$ Aix Marseille Université, CNRS, Centrale Marseille, Institut Fresnel, UMR 7249, 13397 Marseille Cedex 20, France \\ ${ }^{2}$ The Institute of Optics and the Center for Coherence and Quantum Optics, University of Rochester, Rochester, New York 14627, USA \\ ${ }^{3} e$-mail: miguel.alonso@fresnel.fr \\ ${ }^{*}$ Corresponding author: rodrigo.gutierrez-cuevas@fresnel.fr
}

Received 25 August 2020; revised 13 October 2020; accepted 9 November 2020; posted 9 November 2020 (Doc. ID 405988 ); published 14 December 2020

\begin{abstract}
A class of self-similar beams, the Platonic Gaussian beams, is introduced by using the vertices of the Platonic solids in a Majorana representation. Different orientations of the solids correspond to beams with different profiles connected through astigmatic transformations. The rotational symmetries of the Platonic solids translate into invariance to specific optical transformations. While these beams can be considered as "the least ray-like" for their given total order, a ray-based description still offers insight into their distribution and their transformation properties. () 2020 Optical Society of America under the terms of the OSA Open Access Publishing Agreement
\end{abstract}

https://doi.org/10.1364/OL.405988

Structured Gaussian (SG) beams are monochromatic paraxial beams that preserve their transverse intensity profile under propagation up to a uniform scaling. At their waist plane, they can be expressed as a Gaussian multiplied by an appropriate polynomial of the transverse coordinates. They include the Laguerre-Gaussian (LG) and Hermite-Gaussian (HG) beams [1] (separable in polar and Cartesian coordinates, respectively) used in many applications [2-5]. A subclass of SG beams that includes both LG and HG beams are the so-called generalized Hermite-LG (HLG) beams [6-8], which can be generated from either HG or LG beams through what is known as astigmatic transformations, implementable with combinations of cylindrical lenses [9-11] or spatial light modulators mimicking them [12-14]. A HLG beam can be represented as a point on the surface of a modal Poincaré sphere [15-17], for which the two poles correspond to LG beams with opposite orbital angular momenta, and equatorial points correspond to HG beams with different orientations. This sphere representation highlights the group properties of HLG beams under astigmatic transformations (which correspond to rotations of the sphere) and the fact that they accumulate geometric phases under sequences of these transformations $[13,16,18,19]$.

SG beams that are not HLG beams cannot be represented as one point on the sphere. By using Majorana's representation for spin systems $[14,20]$, SG beams can instead be represented by a collection of $N$ points (called "stars") over the sphere, referred to as the Majorana constellation (MC), where $N$ is the order of the polynomial that multiplies the Gaussian. Majorana's representation can be applied to $S G$ beams due to their mathematical analogy with spin systems $[14,16,21-24]$. The MC provides information as to whether a beam is invariant under specific astigmatic transformations through its rotational symmetries. Moreover, these symmetries give way to (continuous or quantized) geometric phases that can be determined solely from the MC [14]. HLG beams are the special cases for which all stars either coincide or are split between one point and its antipode.

For quantum systems, the problem of finding the most symmetric distribution of $N$ points on the sphere has been linked to the search for the "most quantum" (and most entangled) or "least classical" states [25-28]. The solutions depend on the cost function used to quantify symmetry (c.f. [25-30]), but five configurations are always present: the vertices of the Platonic solids (Fig. 1) which also play a role in quantum metrology $[31,32]$ and polarization reconstruction $[33,34]$. In this Letter, we consider the beams resulting from using these five MC distributions, and show that their rotational symmetries lead to beams that are invariant under several astigmatic transformations. In analogy with spin systems, these beams can be considered as the "least ray-like" beams for a given order $N$. However, we show that the ray model still provides insights into their spatial distribution and their changes under astigmatic transformations.

$S G$ beams are mathematically analogous to eigenstates of spin systems [16,22-24,35-37] and can thus be studied through the same operator formalism. However, for the purposes of the current work, it is sufficient to highlight the role of the fields that are analogous to the coherent states for quantum systems [38]. These "coherent state" beams correspond to the extremal HLG beams for which all stars coincide at a point with colatitude $\theta$ and longitude $\phi$ over the sphere. They can be represented by a simple expression in terms of an $N$ th-order Hermite polynomial, $H_{N}$, evaluated at a complex value $[7,8,39,40]$ :

$$
U_{N}\left(\mathbf{r} ; \mathbf{v}_{j}\right)=\frac{(\mathbf{v} \cdot \mathbf{v})^{N / 2}}{w \sqrt{2^{N-1} \pi N !}} e^{-\frac{r^{2}}{w^{2}}} H_{N}\left(\frac{\sqrt{2} \mathbf{v} \cdot \mathbf{r}}{w \sqrt{\mathbf{v} \cdot \mathbf{v}}}\right),
$$

where $w$ is the width of the fundamental Gaussian, and $\mathbf{v}$ is a Jones vector (borrowing the term from polarization), connected to a point $(\theta, \phi)$ over the sphere according to

$$
\mathbf{v}=\left[\cos (\theta / 2) e^{-\mathrm{i} \frac{\phi}{2}} \boldsymbol{\epsilon}_{+}+\sin (\theta / 2) e^{\mathrm{i} \frac{\phi}{2}} \boldsymbol{\epsilon}_{-}\right] e^{-\mathrm{i} \chi / 2},
$$




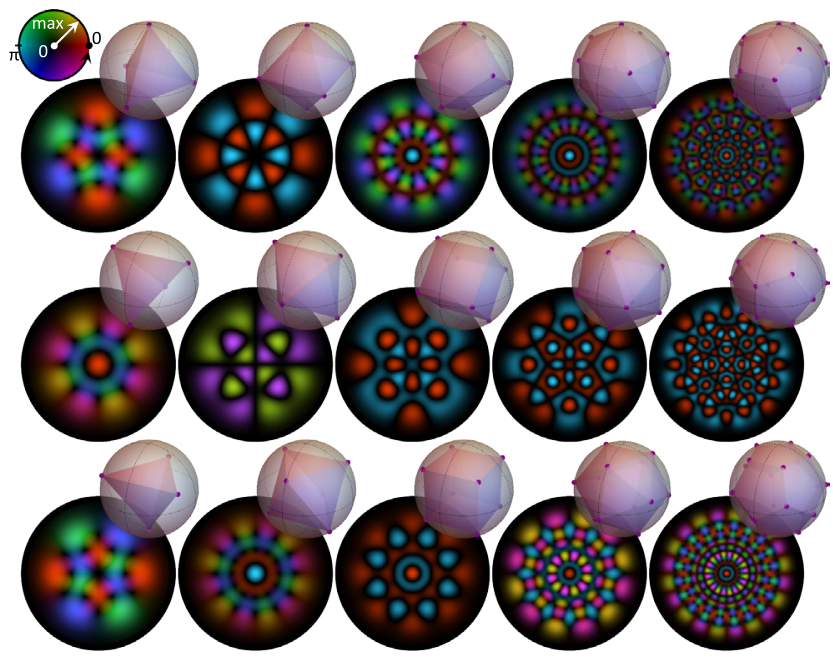

Fig. 1. MC and transverse field with phase encoded as hue for the PG beams. From left to right: tetrahedron (four vertices), octahedron (six vertices), cube (eight vertices), icosahedron (12 vertices), and dodecahedron (20 vertices). Each row shows a different orientation; from top to bottom: vertex, edge, and face upward.

with $\boldsymbol{\epsilon}_{ \pm}=(\widehat{\mathbf{x}} \pm \widehat{\mathbf{y}}) / 2^{1 / 2}$ and $\chi$ controlling the global phase. Let us represent the coherent states by the ket $|N ; \mathbf{v}\rangle$. The Q (or Husimi) function for a generic $S G$ beam $|U\rangle$ is then defined as

$$
Q(\theta, \phi)=\frac{N+1}{4 \pi}|\langle N ; \mathbf{v} \mid U\rangle|^{2},
$$

where the inner product corresponds to integration over the waist plane. The $\mathrm{Q}$ function is a non-negative function over the sphere that is uniquely determined by its $N$ zeros, which correspond precisely to the MC. Astigmatic transformations cause a rotation of the $\mathrm{Q}$ function and hence of the $\mathrm{MC}[14,20]$. Note that the order $N$ of the SG beam must be the same as that for the coherent states; otherwise $N$ is identically zero. A general $N$ th-order SG beam $|U\rangle$ can be expressed as a superposition of, e.g., LG beams of order $N$ according to $|U\rangle=\sum_{\ell} c_{\ell}|N, \ell\rangle$, where $|N, \ell\rangle$ represents a $L G$ beam with vorticity $\ell$.

The vertices of the five Platonic solids can be used as MCs to define the Platonic Gaussian (PG) beams. Figure 1 shows these beams for several orientations of the MC, and Fig. 2 shows that the $\mathrm{MC}$ coincides with the zeros of the $\mathrm{Q}$ function. Note that each of these MCs defines a family of beams since different orientations correspond to different beam shapes. In particular, given the expansion coefficients for the PG beams in terms of LG beams for a given orientation (e.g., those in Table S1 of Supplement 1), those for any other orientation can be obtained (as discussed in [14] and Supplement 1). Due to their uniformly distributed MC, PG beams carry no net orbital angular momentum, even though they often display phase vortices depending on the orientation of the MC, as can be seen in Fig. 1 .

The rotational symmetries of the MC indicate invariance against specific astigmatic transformations. Symmetries of the MC around the vertical axis $(\theta=0$ or $\pi)$ correspond to rotational symmetries of the transverse field distribution, while symmetries along other directions are not obvious from the beam's cross section, as illustrated in Fig. 1 where the MCs are oriented to show different rotational symmetries along the vertical. The PG beams have the most axes of symmetry for

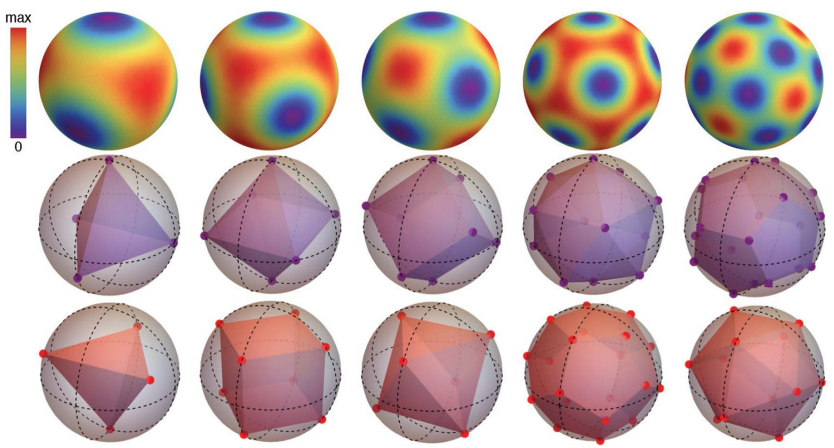

Fig. 2. Q function, $M C$, and $D C$ for the five $P G$ beams given the orientation of the MC in the top row of Fig. 1.

their corresponding order. This does not necessarily mean that they have the most symmetries; HLG beams have a continuous symmetry around a single axis. Notice that, by rotating the MC with combinations of cylindrical lenses, the beam profiles can change from, say, six-fold to four-fold rotational symmetry (in the same way as a HG beam can be transformed into a LG beam by similar means $[9,10])$. Note also that the five Platonic solids can be classified into three categories determined by their symmetry group. The pairs of solids belonging to each group are said to be dual to each other: each has the same number of faces as the other has vertices [41].

The ray model provides insight into the structure of optical beams $[13,17,40,42]$. Typically, a beam is associated with a twoparameter family of rays. For SG beams, this association can be represented as a path over a ray Poincaré sphere, where each point of the sphere corresponds to a one-parameter elliptic ray family generating a ruled hyperboloid upon propagation [40]. At the waist plane, the transverse positions $\mathbf{Q}$ and transverse direction cosine vectors $\mathbf{P}$ of all the rays in this family describe ellipses that can be written in terms of the Jones vector as

$$
\frac{1}{w} \mathbf{Q}+\mathrm{i} \frac{k w}{2} \mathbf{P}=\sqrt{N+1} \mathbf{v} e^{-\mathrm{i} \tau},
$$

where $k$ is the wavenumber and $\tau \in[0,2 \pi]$ is the parameter labeling each ray. The coherent states in Eq. (1) are the fields that most resemble these elliptic ray families, since their intensity profiles outline ellipses [14,40]. A ray-based field estimate then results from dressing the elliptic ray families with the corresponding coherent states while taking into account their phase relation provided by the differences in optical path length. This approach is well suited for beams whose $\mathrm{Q}$ functions show a clear ridge that mimics the ray-based path [40]. This is the case for HLG beams, where the path is a circle and this construction gives the exact field $[8,24,40]$.

As shown in Fig. 2, the Q functions of the PG beams do not form a ridge along a single path; instead, they present isolated maxima distributed uniformly over the sphere at locations corresponding to the centers of the facets of the solid, and therefore to the vertices of the dual solid. These points are referred to here as the dual constellation (DC). PG beams are then the least "ray-like" in the sense that, for a given order $N$, they are the furthest from having a $Q$ function with maxima following a single path over the sphere, and hence of being associated with a two-parameter ray family [25-28]. However, a useful ray picture for PG beams results from associating them with a discrete set 

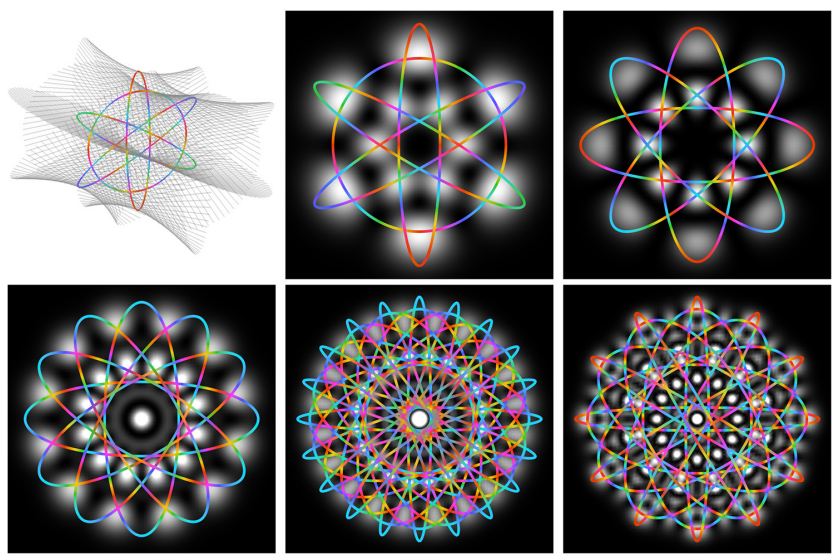

Fig. 3. Propagation of the elliptic ray families associated with the DC for a tetrahedron PG beam and the transverse intensity for the five PG beams given the MC orientation in the top row of Fig. 1, with the elliptic ray families superimposed, with phase encoded as hue. Visualization 1 shows this construction for varying MC orientations.

of one-parameter elliptic ray families, each corresponding to a point of the DC. These ray families are shown in Fig. 3 for the five PG beams with the MC orientations used in Fig. 2, and Visualization 1 shows the variation of the beams and the elliptic ray families for varying orientations of the MCs. It can be seen that the shape outlined by the ray ellipses provides a general idea of the intensity distribution of the corresponding beam.

We now show that ray-based estimates in which each ray family is dressed with a coherent state can also be applied to PG beams. The expression takes the form of a discrete sum:

$$
U_{\mathrm{PG}}(\mathbf{r}) \propto \sum_{j=1}^{n_{\mathrm{d}}} U_{N}\left(\mathbf{r} ; \mathbf{v}_{j}\right),
$$

where each Jones vector $\mathbf{v}_{j}$ corresponds to a DC point. The global phases $-\chi_{j} / 2$ of each Jones vector must be chosen to ensure the self-consistency of this construction. A criterion for assigning these phases emerges from noticing that the overlap between two coherent states gives simply the $\mathrm{Nth}$ power of the inner product of their Jones vectors, namely, $\left\langle N ; \mathbf{v}_{1} \mid N ; \mathbf{v}_{2}\right\rangle=\left(\mathbf{v}_{1}^{*} \cdot \mathbf{v}_{2}\right)^{N}$. We can then choose each $\chi_{j}$ so that this inner product depends only on the relative position of the corresponding two points within the DC. This is achieved by ensuring that, for any pair of first neighbors, $\left(\mathbf{v}_{i}^{*} \cdot \mathbf{v}_{j}\right)^{N}$ is real and positive. If all the corners of a facet, $\chi_{j}$ are chosen sequentially so that $\mathbf{v}_{i}^{*} \cdot \mathbf{v}_{j}$ is real and positive, the phase of the corresponding inner product for the first and last points then equals the accumulated geometric phase [18] given by one half of the solid angle occupied by this facet, namely, $2 \pi / N$ (since the number of facets of the DC equals the number of stars in the $\mathrm{MC})$. When raised to the power $N$, this inner product yields a real and positive number, ensuring the self-consistency of the procedure, which is then extended by moving onto other facets. The resulting sets of Jones vectors for a particular orientation the five Platonic solids are given in Supplement 1. Other orientations of the solids can be found through rotations of the sphere, achieved by multiplying all $\mathbf{v}_{j}$ by a unitary $2 \times 2$ matrix (in analogy with the effect of waveplates for polarization [43]): for a rotation by an angle $\alpha$ around an axis defined by $(\bar{\theta}, \bar{\phi})$, the
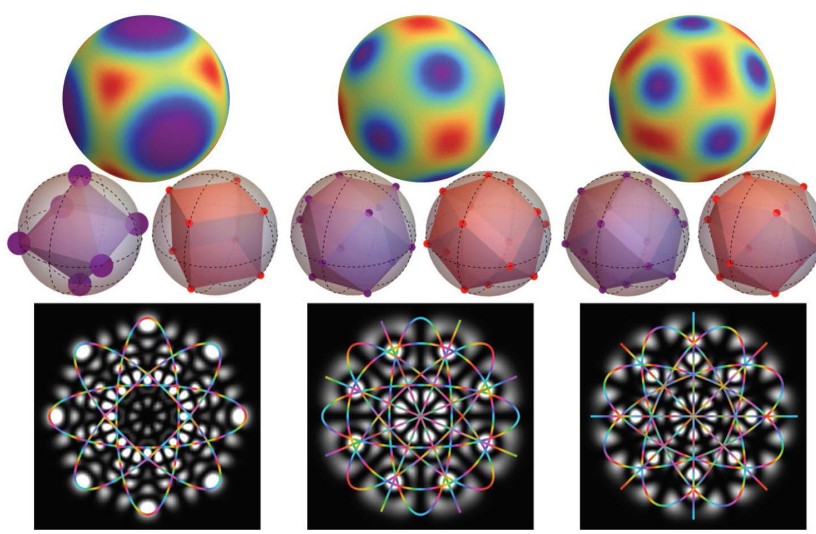

Fig. 4. Higher-order PG beams: (top) Q function, (middle) MC and DC, and (bottom) intensity with elliptic ray families superposed, for (left to right) octahedron with three stars per vertex, cuboctahedron, and spherical projection of the rhombic dodecahedron (the last two being duals).

matrix takes the form $\mathbb{J}=\overline{\mathbf{v}}_{1} \overline{\mathbf{v}}_{1}^{\dagger} e^{-\mathrm{i} \alpha / 2}+\overline{\mathbf{v}}_{2} \overline{\mathbf{v}}_{2}^{\dagger} e^{\mathrm{i} \alpha / 2}$, where the eigenvectors of this matrix, $\overline{\mathbf{v}}_{1,2}$, are given by Eq. (2) evaluated at $(\bar{\theta}, \bar{\phi})$ and $(\pi-\bar{\theta}, \pi+\bar{\phi})$, respectively. In the ray picture, these rotations are performed by $4 \times 4$ symplectic matrices acting on the four-vector $(\mathbf{Q}, \mathbf{P})[14,19]$.

The self-consistency of this construction can be given a ray-based interpretation. Going around an elliptic ray family over the waist plane, given their skewness, an optical path length is accumulated, which we choose as equal to $N+1$ wavelengths. The "phase" assigned to each ray is given by its optical path length times the wavenumber, plus a semiclassical correction of $-\tau$ [40]. This phase is represented in Fig. 3 by the color variations along the ellipses. Note that, in general, the crossings between ellipses where the phases are similar tend to be near intensity maxima, while out-of-phase crossings tend to be near minima (although the extension of the coherent states beyond the ellipses causes interference with nearby ellipses when $N$ is small or the density of crossings is high). As shown in Supplement 1, the locations of these crossings can be obtained via a discrete version of the construction in [40].

Let us now discuss possible extensions to higher orders. Grouping the Platonic solids by their symmetry structure suggests that the cube and dodecahedron can be considered as higher-order PG beams since they have the same symmetries as the octahedron and icosahedron, respectively, although they involve more stars (i.e., higher $N$ ). Higher-order PG beams can also be achieved through MCs that belong to the same symmetry group as the Platonic solids but that involve more stars, say, by simply having multiple stars at each location or by considering the vertices of other solids with the same symmetry structure (e.g., the Archimedean and Catalan solids). Examples of these two types of higher-order PG beams are shown in Fig. 4. Of course, these higher-order beams are not necessarily the most symmetric for the corresponding total order $N$.

A DC can be assigned to these higher-order MCs by using the Q function's maxima, which generally correspond to the dual solid's vertices. The elliptic ray families associated with the DC can still be used to obtain a simple semiclassical estimate:

$$
U_{\mathrm{PG}}(\mathbf{r}) \propto \sum_{j=1}^{n_{\mathrm{d}}}\left\langle N ; \mathbf{v}_{j} \mid U_{\mathrm{PG}}\right\rangle U_{N}\left(\mathbf{r} ; \mathbf{v}_{j}\right) .
$$




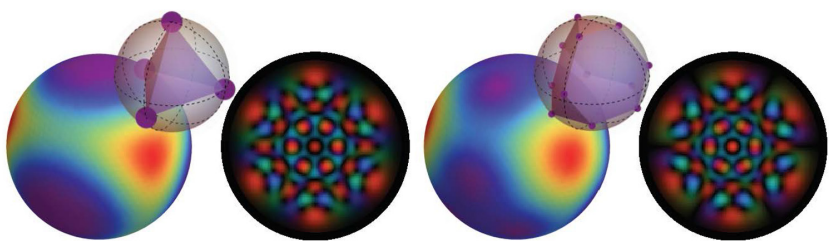

Fig.5. Q function, MC, and field distribution for (left) tetrahedron with three stars per vertex and (right) estimate provided by Eq. (6).

While this equation resembles an orthogonal basis expansion, these coherent states do not form an orthogonal basis, and the sum might involve more or fewer elements than $N+1$, the dimensionality of the Hilbert space. The coefficient $\left\langle N ; \mathbf{v}_{j} \mid U_{\mathrm{PG}}\right\rangle$ in Eq. (6) is a positive real number assigning the appropriate weight to each of the coherent states. These coefficients are all identical for the basic PG beams, but can take different values for beams with a MC (DC) that is not face (vertex) invariant, such as the cuboctahedron beam shown in Fig. 4. The phase relation between the different coherent states is again encoded in the choice of Jones vectors. Through a construction similar to that used for the basic PG beams, the phases $-\chi_{j} / 2$ are chosen so that the phase difference incurred after a cyclic path along neighbors connected by a ridge of the $\mathrm{Q}$ function is equal to zero $\bmod (2 \pi / N)$. If the $\mathrm{DC}$ is face invariant, this reduces to the condition that the Jones vector must chosen so that $\left(\mathbf{v}_{i}^{*} \cdot \mathbf{v}_{j}\right)^{N}$ is real and positive. However, when the DC has different facet shapes, an extra phase must be included to compensate for the geometric phase difference incurred by traveling along adjacent facets subtending different solid angles. Note that for basic PG beams, Eq. (6) is exact and reduces to Eq. (5), while for higher-order beams, it is generally not exact although it provides accurate estimates with the same symmetry structure. This slight discrepancy can be appreciated from the MC shown in Fig. 5 for a tetrahedron PG beam with three stars per vertex.

In conclusion, PG beams are defined by identifying the vertices of the five Platonic solids with the MC. The many symmetries of these solids translate into invariance of the beams under several types of astigmatic transformations. While these beams can be considered as the least ray-like for their order $N$, ray optics still provides insight into their spatial structure. Moreover, it is possible to obtain a wave estimate by using solely information from the discrete ray structure associated with the DC. Quantum states with highly symmetric MCs have found useful applications in metrology [32], so it will be interesting to investigate whether the analogous classical beams described here have analogous metrology applications.

Funding. Excellence Initiative of Aix-Marseille Université A*MIDEX, a French "Investissements d'Avenir" programme.

Acknowledgment. The authors acknowledge M. R. Dennis and L. L. Sánchez-Soto for useful discussions.

Disclosures. The authors declare no conflicts of interest.

See Supplement 1 for supporting content.
2. G. C. G. Berkhout, M. P. J. Lavery, J. Courtial, M. W. Beijersbergen, and M. J. Padgett, Phys. Rev. Lett. 105, 153601 (2010).

3. Y. Zhou, M. Mirhosseini, D. Fu, J. Zhao, S. M. H. Rafsanjani, A. E. Willner, and R. W. Boyd, Phys. Rev. Lett. 119, 263602 (2017).

4. X. Gu, M. Krenn, M. Erhard, and A. Zeilinger, Phys. Rev. Lett. 120, 103601 (2018).

5. M. Tsang, R. Nair, and X.-M. Lu, Phys. Rev. X 6, 031033 (2016).

6. E. G. Abramochkin and V. G. Volostnikov, J. Opt. A 6, S157 (2004).

7. E. G. Abramochkin and V. G. Volostnikov, Phys. Wave Phenom. 18, 14 (2010)

8. R. Gutiérrez-Cuevas, M. R. Dennis, and M. A. Alonso, J. Opt. 21, 084001 (2019).

9. E. Abramochkin and V. Volostnikov, Opt. Commun. 83, 123 (1991).

10. L. Allen, M. W. Beijersbergen, R. J. C. Spreeuw, and J. P. Woerdman, Phys. Rev. A 45, 8185 (1992).

11. J. A. Rodrigo, T. Alieva, and M. L. Calvo, J. Opt. Soc. Am. A 23, 2494 (2006).

12. J. A. Rodrigo, T. Alieva, and M. L. Calvo, Opt. Express 17, 4976 (2009).

13. T. Malhotra, R. Gutiérrez-Cuevas, J. Hassett, M. Dennis, A. Vamivakas, and M. Alonso, Phys. Rev. Lett. 120, 233602 (2018).

14. R. Gutiérrez-Cuevas, S. Wadood, A. Vamivakas, and M. Alonso, Phys. Rev. Lett. 125, 123903 (2020).

15. M. J. Padgett and J. Courtial, Opt. Lett. 24, 430 (1999).

16. G. F. Calvo, Opt. Lett. 30, 1207 (2005).

17. Y. Shen, Z. Wang, X. Fu, D. Naidoo, and A. Forbes, Phys. Rev. A 102, 031501 (2020).

18. M. Berry, J. Mod. Opt. 34, 1401 (1987).

19. T. Alieva and M. J. Bastiaans, Opt. Lett. 32, 1226 (2007).

20. E. Majorana, II Nuovo Cimento 9, 43 (1932).

21. S. van Enk, Opt. Commun. 102, 59 (1993).

22. S. J. M. Habraken and G. Nienhuis, Opt. Lett. 35, 3535 (2010).

23. M. R. Dennis and M. A. Alonso, Phil. Trans. R. Soc. A 375, 20150441 (2017).

24. M. R. Dennis and M. A. Alonso, J. Phys. Photon. 1, 025003 (2019).

25. M. Aulbach, D. Markham, and M. Murao, New J. Phys. 12, 073025 (2010).

26. O. Giraud, P. Braun, and D. Braun, New J. Phys. 12, 063005 (2010).

27. J. Martin, O. Giraud, P. A. Braun, D. Braun, and T. Bastin, Phys. Rev. A 81, 062347 (2010).

28. G. Björk, A. B. Klimov, P. de la Hoz, M. Grassl, G. Leuchs, and L. L. Sánchez-Soto, Phys. Rev. A 92, 031801 (2015).

29. J. H. Conway, R. H. Hardin, and N. J. A. Sloane, Exp. Math. 5, 139 (1996).

30. E. B. Saff and A. B. J. Kuijlaars, Math. Intell. 19, 5 (1997).

31. P. Kolenderski and R. Demkowicz-Dobrzanski, Phys. Rev. A 78 052333 (2008).

32. F. Bouchard, P. de la Hoz, G. Björk, R. W. Boyd, M. Grassl, Z. Hradil, E. Karimi, A. B. Klimov, G. Leuchs, J. Řeháček, and L. L. Sánchez-Soto, Optica 4, 1429 (2017).

33. A. Peinado, A. Lizana, J. Vidal, C. lemmi, and J. Campos, Opt. Express 18, 9815 (2010).

34. M. R. Foreman, A. Favaro, and A. Aiello, Phys. Rev. Lett. 115, 263901 (2015).

35. S. Danakas and P. K. Aravind, Phys. Rev. A 45, 1973 (1992).

36. S. van Enk and G. Nienhuis, Opt. Commun. 94, 147 (1992).

37. G. Nienhuis and L. Allen, Phys. Rev. A 48, 656 (1993).

38. A. M. Perelomov, Commun. Math. Phys. 26, 222 (1972).

39. V. V. Kotlyar, A. A. Kovalev, and A. P. Porfirev, Opt. Lett. 40, 701 (2015).

40. M. A. Alonso and M. R. Dennis, Optica 4, 476 (2017).

41. M. Hamermesh, Group Theory and Its Application to Physical Problems (Dover Books on Physics) (Dover Publications, 1989).

42. Y. Shen, X. Yang, D. Naidoo, X. Fu, and A. Forbes, Optica 7, 820 (2020).

43. A. Vella and M. A. Alonso, Opt. Lett. 43, 379 (2018).

\section{REFERENCES}

